chronic inflammation. These factors induce alterations in several cell types in the heart.

In HFpEF, the endothelial cells that line small blood vessels in the heart become dysfunctional and fail to synthesize adequate amounts of nitric oxide (NO), a substance involved in the relaxation of blood vessels. Connective-tissue cells called fibroblasts generate scarring. And the cardiomyocytes themselves become stiffer because of changes related to proteins involved in molecular signalling pathways and cellular contraction ${ }^{5}$. The end result is sluggish filling of the heart.

None of the existing therapies for HFrEF helps in HFpEF, and a good animal model for HFpEF has been lacking. Modelling HFpEF is challenging because of the complex interplay of many contributing factors and the plethora of clinical manifestations of the disease. Rather than recreating all the stimuli that lead to HFpEF, Schiattarella et al. combined two major risk factors - obesity linked with glucose intolerance (an inability to transport sugar efficiently from the blood into cells, which can lead to diabetes) and high blood pressure - in mice. The authors' reasoning was that each of these factors activates several disease-associated pathways.

Mice were rendered obese and glucoseintolerant with a high-fat diet. High blood pressure was induced by administration of a drug called $N^{\omega}$-nitro-L-arginine methyl ester (L-NAME), which inhibits the enzymes that are normally expressed at constant levels to catalyse the production of $\mathrm{NO}$ (called constitutive NO synthases). Mice treated in this way had several characteristics of HFpEF, including impaired filling of the heart, inability to exercise, lung congestion and increased levels of molecular markers of inflammation in the heart and blood (Fig. 1). Notably, ejection fraction in these mice remained normal up to at least 12 months of age, which is half the lifespan of a mouse.

The authors then used their model to investigate the role of misfolded proteins in HFpEF. These proteins accumulate in several disorders in which cardiac stress is present ${ }^{6}$, including HFpEF, and they activate a molecular program known as the unfolded protein response (UPR $)^{7}$. Schiattarella et al. focused on inositol-requiring enzyme $1 \alpha$ (IRE1 $\alpha)$, which is activated in response to cellular stress caused by an excess of misfolded proteins. IRE1 $\alpha$ cuts the messenger RNA that encodes X-box binding protein 1 (XBP1) and transforms it into a shorter mRNA that encodes a protein called $\mathrm{XBP} 1$ spliced (XBP1s). XBP1s is a transcription factor that activates genes involved in the UPR.

Schiattarella and colleagues found that IRE $1 \alpha$ activation and XBP 1 s levels were reduced in the hearts of HFpEF model mice, as well as in heart-tissue samples from people with HFpEF, when compared with those from healthy individuals (Fig. 1). By contrast, cardiac XBP1s levels were increased or unchanged in mice and humans with HFrEF. The decrease

in XBP1s levels in the hearts of HFpEF mice was due to IRE1 a modification by $S$-nitrosylation. This process, in which an NO molecule is attached to specific sulfur atoms on proteins, is known to decrease IRE1 $\alpha$ activity ${ }^{8}$.

Although Schiattarella et al. did not identify the cells that produce $\mathrm{NO}$, they observed that inducible NO synthase (iNOS), an enzyme that was highly expressed in their HFpEF model, promoted IRE1 a S-nitrosylation. They

\section{"The work} demonstrates that two commonrisk factors are sufficient to reproduce many of the manifestations of this disease" went on to show that pharmacological or genetic inhibition of iNOS or cardiomyocyte-specific overexpression of XBP1s attenuated cardiac filling defects, the inability to exercise, and lung congestion in mice induced to develop features of HFpEF. These findings highlight the role of XBP1s loss in the mechanisms underlying this disease.

This work demonstrates that two common risk factors for HFpEF are sufficient to reproduce many of the manifestations of this disease. The authors' mouse model will be useful for dissecting disease mechanisms and developing new treatments. Although large-animal models are often valuable in studying complex physiological processes and testing therapeutics, the mouse has the advantage of being easy to manipulate genetically and having a short lifespan ${ }^{9}$. The latter factor will also facilitate the study of the influence of ageing, another key risk factor for HFpEF.

Some questions remain unanswered. The less-than-complete reversal of HFpEF disease manifestations observed in response to iNOS inhibition suggests that other molecular pathways are also involved in the reduction of XBP1s levels. More generally, the study explored a single candidate mechanism for this disease. It is likely that other mechanisms also contribute, and the new HFpEF mouse model provides an in vivo platform with which to define them.

\section{Dulguun Amgalan and Richard N.}

Kitsis are in the Departments of Medicine (Cardiology) and Cell Biology, and in the Wilf Family Cardiovascular Research Institute, Albert Einstein College of Medicine, Bronx, New York 10461, USA.

e-mail:richard.kitsis@einstein.yu.edu

1. Savarese, G. \& Lund, L. H. Card. Fail. Rev. 3, 7-11 (2017).

2. Schiattarella, G. G. et al. Nature 568, 351-356 (2019).

3. Bloom, M. W. et al. Nature Rev. Dis. Primers 3, 17058 (2017).

4. Dunlay, S. M., Roger, V. L. \& Redfield, M. M. Nature Rev. Cardiol. 14, 591-602 (2017).

5. Shah, S. J. et al. Circulation 134, 73-90 (2016)

6. Wang, Z. V. \& Hill, J. A. Cell Metab. 21, 215-226 (2015).

7. Walter, P. \& Ron, D. Science 334, 1081-1086 (2011).

8. Yang, L. et al. Science 349, 500-506 (2015).

9. Valero-Muñoz, M., Backman, W. \& Sam, F. J. Am. Coll. Cardiol. Basic Transl. Sci. 2, 770-789 (2017).

This article was published online on 10 April 2019.

\title{
ENERGY STORAGE
}

\section{Accurate predictions of lithium-ion battery life}

Highly reliable methods for predicting battery lives are needed to develop safe, long-lasting battery systems. Accurate predictive models have been developed using data collected from batteries early in their lifetime.

\section{MAITANE BERECIBAR}

A problem that hinders the rapid development of battery technology is that testing and monitoring battery 'health' - which affects battery life - takes a long time. Better methods for predicting battery lives are, therefore, much needed, but are extremely challenging to develop. Writing in Nature Energy, Severson et al. ${ }^{1}$ report that machine learning can be used to construct models that accurately predict battery lives, using data collected from charge-discharge cycles measured in the early stages of a battery's life.

Society needs to stop producing carbon emissions if it is to tackle the impending climate crisis. To achieve this aim, a twofold approach has emerged: the electricity sector is making use of renewable-energy sources; and electric vehicles are replacing those that use conventional combustion engines. Both transitions come with their own barriers.

One of the main hurdles for renewable energy is that the sources are often intermittent, producing an imbalance between demand and supply. The issues with electrified transport are ensuring that enough electricity is produced to charge all vehicles when conventional combustion engines are no longer used, and the integration of charging infrastructure with the electrical grid. The on-board 
energy-storage units of electric vehicles could, in principle, act as an energy buffer for the electrical grid if bidirectional chargers are deployed - cars spend most of their time parked, so by returning their electricity to the grid when stationary, they could help to compensate for fluctuations in the supply associated with renewable energy. The commercialization of electric and hybrid vehicles has, therefore, stimulated a growing demand for long-lived batteries, both for driving and for grid buffering. As a result, methods for assessing battery health are becoming increasingly important.

A figure of merit known as state of health $(\mathrm{SOH})$ is used to quantify the ability of a battery to store energy, relative to its initial or ideal conditions. $\mathrm{SOH}$ is reported as a percentage: it is $100 \%$ when the battery is new, but decreases over time. Estimating the $\mathrm{SOH}$ of a battery is essential to ensure safe and correct usage. However, there is no consensus in industry or among scientists about what exactly $\mathrm{SOH}$ is, or on how it should be determined.

The $\mathrm{SOH}$ of a battery reflects two phenomena that occur as batteries age: progressive loss of storage capacity, which is known as capacity fade; and progressive increase of impedance (a measure of electrical resistance), which causes the power provided by the battery to decline. Estimates of a battery's $\mathrm{SOH}$, therefore, have to take into consideration both capacity fade and impedance increase.

Unfortunately, lithium-ion batteries are complex systems, which means that capacity fade and impedance increase arise from several interacting processes. Most of these processes cannot be studied independently and occur at similar timescales, which complicates the investigation of ageing mechanisms. $\mathrm{SOH}$, therefore, cannot be determined from a single direct measurement. Conventional methods for estimating $\mathrm{SOH}$ involve studying interactions between a battery's electrodes (see ref. 2, for example). Unfortunately, most of these methods make the battery unusable, which is clearly undesirable.

$\mathrm{SOH}$ can be determined in a less destructive way by using two different approaches ${ }^{3}$ : adaptive models and experimental techniques. Adaptive models learn from battery-performance data and thereby self-adjust to reduce errors. They are useful when system-specific information about a battery is not available, and are good for diagnosing the degradation processes that affect battery health. The main problem with such models is that they need to be trained using experimental data before they can be used to determine the present capacity of a battery.

Experimental techniques can be used to evaluate specific physical processes and failure mechanisms that occur in a battery, enabling the future rate of capacity degradation to be estimated (see ref. 4, for example). A limitation of these approaches is that they cannot detect intermittent failures. Alternative techniques that consider the rate of change of voltage or capacity (rather than raw voltage and current data) have also emerged in the past few years as useful tools for revealing battery-degradation mechanisms ${ }^{5}$. Nevertheless, finding other methods that accurately predict the lifetime of batteries is essential to accelerate the development of battery technology.

Severson et al. have tackled this challenge by generating a comprehensive data set that characterizes the performance of 124 commercial lithium-ion batteries (more specifically, batteries that use lithium iron phosphate as the cathode material and graphite as the anode) as they undergo cycles of charging and discharging. The authors used a variety of fastcharging conditions (but identical discharging conditions) to alter the cycle lives of the batteries - the number of cycles that a battery goes through before it reaches the end of its life. A wide range of cycle lives (from 150 to 2,300) was therefore captured in the data.

The researchers then used machine learning to analyse the data, and thereby produced models that can reliably predict battery cycle lives. Notably, the authors analysed data from only the first 100 cycles of each experimentally characterized battery - which was before the batteries showed clear signs of capacity fade. The best model correctly predicted cycle lives for about $90.9 \%$ of the batteries in the study (Fig. 1). Severson et al. also used their approach to analyse data from just the first five cycles of experimental data to classify batteries as either having a short or a long lifetime (that is, cycle lives of fewer than or more than 550 cycles, respectively). In this case, about $95 \%$ of the model's predictions were correct.

It is notable that this level of accuracy was achieved by analysing data recorded during discharge experiments, rather than by only considering capacity-fade data. Moreover, the models did not involve slow test cycles, or require any assumptions to be made about the chemistry and degradation mechanisms occurring in the batteries, which had been the case in previously reported studies that used machine learning ${ }^{6}$. However, it should be noted that the new models were less effective at estimating cycle lifetimes for batteries that had undergone greater degradation.

Severson and colleagues' work shows that data-driven modelling using machine learning is a promising route for prognostics of lithium-ion batteries, and could, therefore, aid the development, manufacture and optimization of emerging battery technology. The authors propose that their models identify degradation modes that are not manifested in capacity-fade data during early cycles, but are instead evident in plots of voltage data. Accordingly, their approach for predicting cycle life complements all previously used approaches. More broadly, the study reveals the potential of combining deliberately generated data with data-driven modelling to predict the behaviour of complex dynamical systems.

\section{Maitane Berecibar is at the Mobility,} Logistics and Automotive Technology Research Centre (MOBI), Vrije Universiteit Brussel, 1050 Brussels, Belgium.

e-mail:maitane.berecibar@vub.be

1. Severson, K. A. et al. Nature Energy https://doi. org/10.1038/s41560-019-0356-8 (2019).

2. Yi, L. et al. J. Power Sources 373, 40-53 (2018).

3. Berecibar, M. et al. Renew. Sust. Energ. Rev. 56, 572-587 (2016).

4. Dubarry, M. et al. J. Power Sources 360, 59-69 (2017).

5. Dubarry, M., Truchot, C. \& Liaw, B. Y. J. Power Sources 219, 204-216 (2012).

6. Berecibar, M. et al. J. Power Sources 320, 239-250 (2016).

This article was published online on 9 April 2019. 